\section{THE TRANSPLANTATION OF SCA-1+/PW1+/PAX7- SKELETAL MUSCLE-DERIVED INTERSTITIAL PROGENITOR CELLS (PICS) IMPROVES CARDIAC FUNCTION IN MICE SUBJECTED TO MYOCARDIAL INFARCTION}

Prashant Jay Ruchaya*, Sachin Amin, Giulia Gritti, Georgina Ellison-Hughes. King's College London

10.1136/heartjnl-2017-311726.204

Introduction Stem cell-based regenerative therapies are fast becoming an attractive and highly promising treatment for heart disease and failure. Sca- $1^{+} / \mathrm{PW} 1^{+} / \mathrm{Pax} 7^{-}$skeletal musclederived interstitial progenitor cells (PICs) regenerate adult skeletal and smooth muscle. Recently, we showed that PICs can differentiate into cardiomyocyte-like cells in vitro. These findings have subsequently opened the potential for PICs to be used as a regenerative therapy in heart failure..

Aim We sought to investigate the in-vivo physiological effects of transplantation of PICs in mice subjected to myocardial infarction (MI).

Methods Myocardial infarction was induced through the ligation of the left anterior descending coronary artery in 89 week old male C57BL/6 mice. 500,000 Sca- $1^{+} / \mathrm{PW} 1^{+} / \mathrm{Pax}^{-}$ PICs (MI+PICs) or PBS (MI-PBS) were transplanted intramyocardially in two regions of the border zone, immediately after MI induction. SHAM mice underwent the same surgical procedure, without ligating the coronary artery, or the intramyocardial transplantation of PICs or PBS. Echocardiography was performed prior to surgery (baseline), and week 3 post-MI and cell transplantation. Mice were sacrificed at 3 weeks postMI, and histology and Masson Trichrome staining was performed to assess infarct size and level of fibrosis.

Results A significant $(\mathrm{p}<0.05)$ improvement in Ejection Fraction (EF) was observed in MI mice transplanted with PICs (MI-PICs), compared to MI+PBS at week 3 post-MI (55.8 $\pm 4.2 \%$ vs. $35.2 \pm 3.6 \%$, respectively). However, this improvement of $\mathrm{EF}$ in $\mathrm{MI}+\mathrm{PICs}$ remained significantly $(\mathrm{p}<0.05)$ lower, compared to SHAM $(71.1 \pm 0.9 \%)$ at matched time points, and to corresponding baseline $(69.6 \pm 0.7 \%)$ levels. A similar trend in Fractional Shortening (FS) was observed, where the MI+PICs group showed a significant $(\mathrm{p}<0.05)$ improvement, compared to the MI+PBS group $(29.2$ $\pm 2.6 \%$ vs. $17.0 \pm 2.0 \%$, respectively). The improvement in FS remained significantly $(\mathrm{p}<0.05)$ lower in the $\mathrm{MI}+\mathrm{PICs}$ group, compared to SHAM $(38.8 \pm 1.4 \%)$ and to corresponding baseline levels $(38.8 \pm 0.5 \%)$. Infarct size and fibrosis significantly $(\mathrm{p}<0.05)$ decreased in the MI-PICs group $(24.2 \pm 2.2 \%$ of LV), compared to the MI-PBS group $(40.2 \pm 2.6 \%$ of LV).

Conclusions Transplantation of skeletal muscle-derived $\mathrm{Sca}-1^{+} /$ PW $1^{+} / \mathrm{Pax}^{-}$PICs into the myocardial infarcted myocardium improves cardiac function, and reduces infarct size. Although the mechanism of action needs to be clarified, PICs could be a promising stem/progenitor cell type for the treatment of heart failure.

\section{VASCULAR SMOOTH MUSCLE CELL HETEROGENEITY AND PLASTICITY}

${ }^{1}$ Annabel Taylor*, ${ }^{2}$ Lina Dobnikar, ${ }^{2}$ Mikhail Spivakov, ${ }^{1}$ Helle JÃfÂ. Cardiovascular Medicine, University of Cambridge; ${ }^{2}$ Nuclear Dynamics ISP, Babraham Institute

10.1136/heartjnl-2017-311726.205
Introduction Vascular smooth muscle cells (VSMCs) show inherent plasticity, enabling their phenotypic switch into a synthetic state for vascular repair and remodelling. Under inflammatory conditions, this contributes to atherosclerotic plaque development, with VSMCs demonstrated to produce multiple plaque-resident cell types. However, only a small fraction of VSMCs participate in disease associated proliferation ${ }^{1}$ and it is unclear if this is a defined subset within the heterogeneous population.

Methods Here we performed transcriptional profiling of dissociated VSMCs from two discrete aortic regions; the plaquesusceptible aortic arch (AA) and resistant descending thoracic aorta (DT). Differentially expressed genes were identified using both conventional and single cell RNA sequencing. This differential expression was then validated by RT-qPCR and single molecule RNA fluorescence in situ hybridisation (smRNA-FISH), implemented to interrogate candidate gene expression at a single-cell level.

Results RNA sequencing analysis demonstrated differential expression of 227 genes between the AA and DT regions, with consistency in the profiling data from conventional and single cell sequencing. These results correlate with earlier work to profile these regions; for example, we found upregulation of homeobox genes in the DT relative to the AA, which has been previously observed ${ }^{2}$. However, many of the genes identified are distinct from those previously characterised and our single cell analysis demonstrated heterogeneity between individual cells within the same region. In particular, certain genes were highly expressed in only a subset of AA cells, including Pde1c, an enzyme shown to promote VSMC proliferation $^{3}$. Patterns in expression were validated for candidate genes, selected by VSMC or disease relevance. This showed similar fold changes in expression between the two regions by RT-qPCR and RNA sequencing, with ongoing smRNA-FISH investigations into their cell to cell variation.

Conclusions Our characterisation of VSMC expression patterns showed both regional and local heterogeneity and suggested the presence of a subset of AA cells with a unique expression profile. These cells might be those responsible for the clonal proliferation we observed in disease ${ }^{1}$, which would explain the AAs heightened plaque susceptibility. Our identified differentially expressed candidate genes may mark or regulate this population, aiding future investigations into the mechanisms underlying plaque development.

\section{REFERENCES}

1. Chappell, J. et al. Circ Res 2016;119:1313-1323.

2. Trigueros-Motos, L. et al. ATVB 2013;33:1248-1256.

3. Satoh, K. et al. Circ Res 2015;116:1098-1100.

\section{CARDIOPROTECTION BY THE MITOCHONDRIA- TARGETED SUPEROXIDE GENERATOR MITOPARAQUAT IN A MURINE MODEL OF ACUTE MYOCARDIAL ISCHAEMIA REPERFUSION INJURY} ${ }^{3}$ Richard Hartley, ${ }^{2}$ Michael Murphy, ${ }^{1}$ Thomas Krieg. 'Department of Medicine, University of Cambridge; ${ }^{2}$ MRC Mitochondrial Biology Unit; ${ }^{3}$ WestCHEM School of Chemistry, University of Glasgow

\subsection{6/heartjnl-2017-311726.206}

Introduction Myocardial infarction is a major cause of death and disease worldwide. Mitochondrial reactive oxygen species 
(ROS) are known to play a central role in the tissue damage caused by ischaemia-reperfusion injury (IRI), yet the use of antioxidant supplements in large scale clinical trials has been shown to have no beneficial effect and indeed some studies have suggested that the addition of exogenous ROS may decrease infarct size. This suggests that mitochondria may exhibit a biphasic response to ROS, termed mitohormesis, characterised by beneficial effects at low doses and detrimental effects at high doses. To investigate this phenomenon, a tool is required to precisely titrate mitochondrial ROS. MitoParaquat is a novel mitochondria-targeted molecule that redox cycles at complex I to produce superoxide, closely mimicking the production of superoxide as the proximal ROS species in vivo. Here it is used to investigate the role of ROS in protection against acute myocardial IRI.

Methods Male C57BL6/J mice aged 8-10 weeks were administered MitoParaquat or vehicle only control by intravenous injection $15 \mathrm{~min}$ before the induction of $30 \mathrm{~min}$ myocardial ischaemia by ligation of the left anterior descending coronary artery. After 2 hours of reperfusion, infarct size was determined by tripheyltetrazolium chloride staining.

Results MitoParaquat decreased infarct size relative to vehicle only control $(42.3 \pm 4.3 \%)$ at doses of $1 \mathrm{nmol}(30.0 \pm 4.0 \%)$, $100 \mathrm{pmol}(22.7 \pm 2.3 \%)$, and $10 \mathrm{pmol}(24.2 \pm 2.0 \%)$. At 1 pmol, no significant difference from vehicle only control was observed $(41.5 \pm 8.6 \%)$, and at $5 \mathrm{nmol}$ it was found to be lethal. There was no significant difference in the area at risk between any groups.

Conclusions First and foremost, the generation of low doses of exogenous ROS by MitoParaquat is shown to be protective against acute myocardial IRI in vivo. MitoParaquat is shown to exhibit a hormetic dose response curve, with protection conferred only in an intermediate dose range with high doses found to be lethal and infarcts from low dose not significantly different from control. Further work is required to determine the mechanism by which this cardioprotection occurs.

\section{EVALUATION OF ACUTE CARDIAC DAMAGE IN A PORCINE MODEL OF DEFIBRILLATION}

${ }^{1}$ Fiona Rogan*, ${ }^{2}$ Rebecca Funston, ${ }^{1}$ Brian Meenan, ${ }^{1}$ George Burke. ${ }^{1}$ Ulster University; ${ }^{2}$ Heartsine Technologies

\subsection{6/heartjnl-2017-311726.207}

Introduction Transthoracic defibrillation is performed to correct life threatening cardiac arrhythmias of the heart, i.e. ventricular fibrillation (VF) and ventricular tachycardia (VT). These are due to chaotic electrical excitation of the heart chambers and loss of coordinated contraction of myocytes that could induce cardiac arrest. Resuscitation guidelines recommend defined defibrillation energies for patient recovery, research indicates that these techniques may cause myocardial damage. However, there is limited information regarding the defibrillation potentially damaging effects on the tissues of the heart at structural and genomic levels. This study investigates the extent of myocardial injury associated with the use of increasing energies $(75 \mathrm{~J}, 150 \mathrm{~J}, 200 \mathrm{~J}$ and $360 \mathrm{~J}$ ) of defibrillation in a porcine model of cardiac arrest using genomic, histological and ultrastructural analysis techniques.

Methodology General anaesthesia was induced in swine models ( $\tilde{\mathrm{A}} \notin \hat{\mathrm{a}} €{ }^{\circ} \ddot{\mathrm{E}} \dagger 10-40 \mathrm{~kg}$ ) in accordance with The Home Office guidelines. VF was induced and defibrillation administered, each animal receiving 20 shocks at the defibrillation energy protocol at $3 \mathrm{~min}$ intervals followed by 6 hour recovery period. Upon completion, animals were humanely euthanised. Cardiac tissues were excised and processed for genomic, histological and ultrastructural analyses and examination.Results: Haemodynamic results demonstrated ROSC occurred in all pigs. Troponin I levels were elevated 3-4 hours after the completion of defibrillation protocol. Gross pathological examination demonstrated no unusual changes. Histological and SEM analysis indicate defibrillation causes changes to the porcine cardiac tissue as evidenced by instances of hypereosinophilia, increased collagen-I deposition and areas of multifocal acute subendocardial, epicardial and myocardial necrosis. qPCR analysis indicates defibrillation induces genomic changes, there was an upregulation in the mRNA expression of structural and inflammation related genes such as Collagen-I, IL-6/18 and MCP1. Hydroxyproline analysis and SEM imaging also illustrated minor changes in collagen content and structural appearance of the tissue, further supported with Image J colour hue analysis.

Discussion The current paradox is cardiac defibrillation depends on the successful selection of energy to generate sufficient current flow through the heart to achieve defibrillation, whilst causing minimal injury to the heart. At this acute timeframe (post protocol), the animal models illustrated biological effects from repeated defibrillation upon cardiac tissues at a structural and genomic level and suggests there is a cardioprotective measure taken by the cardiomyocytes due to electrical overstimulation. In conclusion, these results show repeated defibrillation at increasing energies produces immediate changes to the functional myocardium at a genomic, microscopic and ultrastructural level.

\section{SYNDECAN-4/FGF-2/PKCA SIGNALLING REGULATES VASCULAR SMOOTH MUSCLE CELL CALCIFICATION VIA CROSS-TALK WITH TGFB}

${ }^{1}$ Samantha Borland*, 'Shona Borland, ${ }^{2}$ Mark Morgan, ${ }^{3}$ Sheila Francis, ${ }^{4}$ Catherine Merry, ${ }^{1}$ Ann Canfield. ${ }^{1}$ University of Manchester; ${ }^{2}$ University of Liverpool; ${ }^{3}$ University of Sheffield; ${ }^{4}$ University of Nottingham

\subsection{6/heartjin-2017-311726.208}

Vascular smooth muscle cells (VSMCs) were induced to mineralise with $\beta$-glycerophosphate ( $\beta$-GP). Controls were cultured without $\beta$-GP. FGF-2 mRNA $(\sim 40$-fold increase, $\mathrm{P}<0.001)$ and protein $(\sim 2$-fold increase, $\mathrm{P}<0.05)$ expression are significantly increased in mineralising VSMCs. FGF-2 also localises to sites of calcification within human atherosclerotic plaques. The expression of syndecan-4, a heparan sulphate proteoglycan which acts as a co-receptor for FGF-2 signalling, is also increased in mineralising VSMCs $(\sim 5$-fold, $\mathrm{P}<0.001)$ and co-localizes with FGF-2 in human calcified atherosclerotic plaques. Exogenous FGF-2 inhibits VSMC mineralisation $(\mathrm{P}<0.001)$ and this inhibition is reduced when syndecan-4 expression is knocked-down using siRNA.

Biochemical inhibition of FGFR signalling using a pan FGFR inhibitor (BGJ398) increases transforming growth factor- $\beta 1$ (TGFß1)-induced Smad2 phosphorylation in VSMCs. As TGF $\beta 1$ increases mineral deposition by VSMCs $(\sim 2$-fold, $\mathrm{P}<0.01)$, the relationship between FGF and TGF $\beta$ signalling in VSMC mineralisation was investigated. Inhibiting FGFR signalling using BGJ398 or knocking-down syndecan-4 expression in VSMCs 